\title{
Enchanting smile with laminate veneers (Contact lens of teeth) - A case report
}

\author{
Humaira Mushtaq ${ }^{*}$, Narendra Kumar ${ }^{2}$ \\ ${ }^{1}$ Post Graduate Student, ${ }^{2} \mathrm{HOD}$, Dept. of Prosthodontics Crown and Bridge, Institute of Dental Studies and Technologies, Ghaziabad, Uttar \\ Pradesh, India \\ *Corresponding Author: Humaira Mushtaq \\ Email: sofihumaira33@gmail.com
}

\begin{abstract}
From a purely cosmetic standpoint, the value of the appearance of one's teeth has taken on greater importance in today's society. Various multiple options are available to serve the problem arising in esthetic zone. Porcelain laminate veneers are considered as most conventional and esthetic restoration that can be used to augment esthetics. The use of laminate veneers has been shown a feasible treatment option in the anterior esthetic zone. This case report discusses a patient having spacing in anterior region with discolored and old composite restorations in interdental region. The patient was then treated with porcelain laminate veneers in the maxillary arch for the same.
\end{abstract}

Keywords: conservative preparation, Esthetic, Etched enamel, laminate veneer, Porcelain.

\section{Introduction}

Confidence is an important part of one's personality and a confident smile makes the picture complete. A shiny smile in accordance with the lips and face adds to the beauty of the person's personality. Dentist's talent, artistry, and expertness in listening to the certain desires of his patient help to design a smile that looks perfect with the face and personality of each individual patient. Laminate veneers are the most consequential and well-accepted restoration in esthetic dentistry which is forged of the chairside composite, porcelain or cast ceramic material. Due to their longevity, aesthetics and conservative nature of veneers, they have been accepted as the most viable treatment modalities since their instigation in $1983 .{ }^{1}$

As being less invasive, for soft and hard tissues and achieving a moderate aesthetic outcome, the treatment procedure with porcelain veneers have received credence by patients. A number of clinical studies have concluded that bonded laminate veneer restorations delivered good results over a period of 10 years and more. ${ }^{2}$ The present case report registers the treatment of spacing and discolored restoration in the anterior esthetic region with thin porcelain laminate veneers, to renovate esthetics and function.

\section{Case Report}

A 26-year-old female patient reported to the Department of Prosthodontics and crown and bridge, IDST, Modinagar with a pivotal complaint of interdental spacing as well as discolored composite restoration in $11,12,13,14,21,22$, 23, 24 and fancied esthetic rehabilitation for the same (Fig. 1). The patient had undergone the procedure of composition restoration for the same problem which has resulted in discoloration after 2 years.

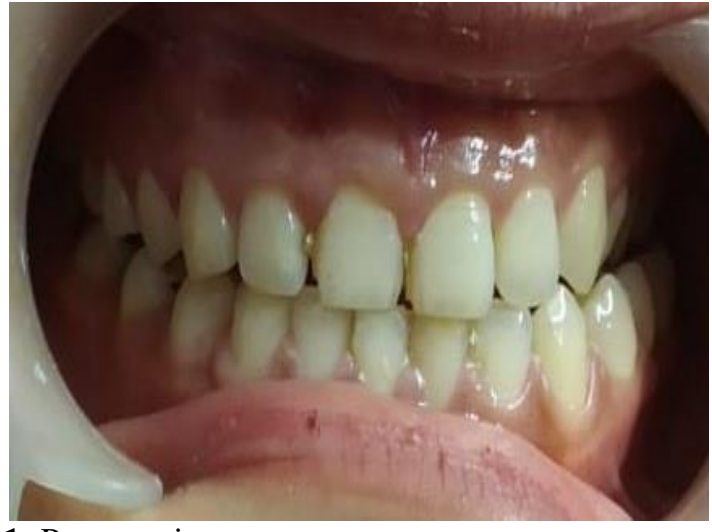

Fig. 1: Preoperative

All teeth were vital and had no hypersensitivity. After examining the radiographic images and digital photographs of the anterior teeth, various treatment options were discussed with the patient which includes laminate veneers, bleaching, and micro abrasion. Contemplating the less invasive nature and astounding esthetic qualities it was concluded to upgrade her appearance using porcelain laminate veneers. From right $1^{\text {st }}$ premolar to left $1^{\text {st }}$ premolar Porcelain laminate veneer was outlined for maxillary arch.

The shade was selected under direct sunlight with VITA 3D master shade guide. Preparation of maxillary and mandibular cast was done. Smile line of the patient was properly analyzed and it was planned to place porcelain veneers from $1^{\text {st }}$ premolar to $1^{\text {st }}$ premolar region in a maxillary arch with incisal overlap preparation. $0.3 \mathrm{~mm}$ and $0.5 \mathrm{~mm}$ three wheel diamond depth-cutting bur were used for placing orientation grooves on a facial surface of tooth respectively.

Tooth preparation was done with a long round ended tapered diamond bur. $0.3 \mathrm{~mm}$ chamfer finish line was prepared in the cervical region and kept subgingivally. Distally the tooth preparation was extended into the contact area (Fig. 2). 


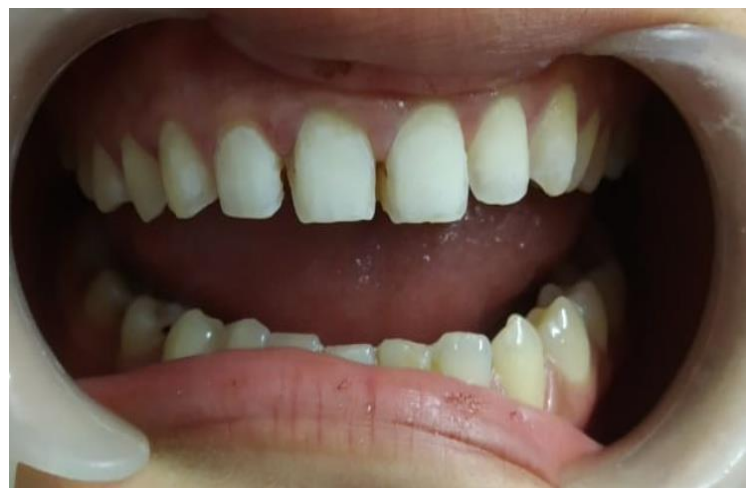

Fig. 2: Teeth preparation done for laminates

Round end tapered diamond bur was used to prepare palatal finish lines with one fourth down the lingual surface and connecting the two proximal finish lines. Sharp round angles and line angles were rounded properly. An impression was prepared with polyvinylsiloxane by putty wash technique after tooth preparation and was then sent to the laboratory (Fig. 3).

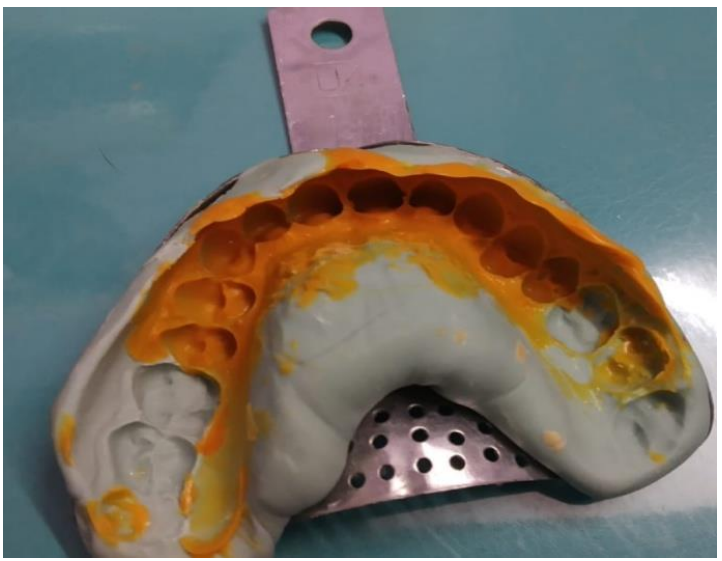

Fig. 3: Impression made with putty light body

Temporization was done for a month. Teeth were cleaned using pumice and were dried after the removal of temporary veneers. The colour, fit and esthetic was checked by placing porcelain veneer IPS-Emax onto tooth with the selected shade of try in a paste. After removal etching was done on the inner side of porcelain veneer with $5 \%$ hydrofluoric acid for 20 seconds, washed under running water and dried. On an inner surface of veneer silane coupling agent (Monoborid-S, IvoclarVivadent) layer was applied and air dried after one minute, the bonding agent was coated and polymerized with a light cure for 60 seconds on the silanized surface.

After that $37 \%$ phosphoric acid was used to etch the prepared tooth for 30 seconds rinsed thoroughly and dried. The bonding agent (Adper single bond 3M ESPE USA) was applied on to the tooth surface and polymerized with a light cure. Dual cure resin cement (Kerr Maxcem Elite) was used to bond the veneer to the tooth.

The resin cement (base and catalyst) after mixing in proportion was applied on veneers and then positioned over the teeth precisely with slight pressure, the excess cement was removed with a brush. Luting cement was polymerized with a light cure for 10 seconds and the veneers were fixed to the teeth. The polymerization was further continued for 60 seconds first from the lingual side and then each segment of veneer was light cured independently for 40 seconds (Fig 4). Occlusion was assessed to ensure that no premature contact existed on tooth-porcelain interfaces. Dental floss was used to check the interproximal contact of each veneer. The patient cherished her new smile.

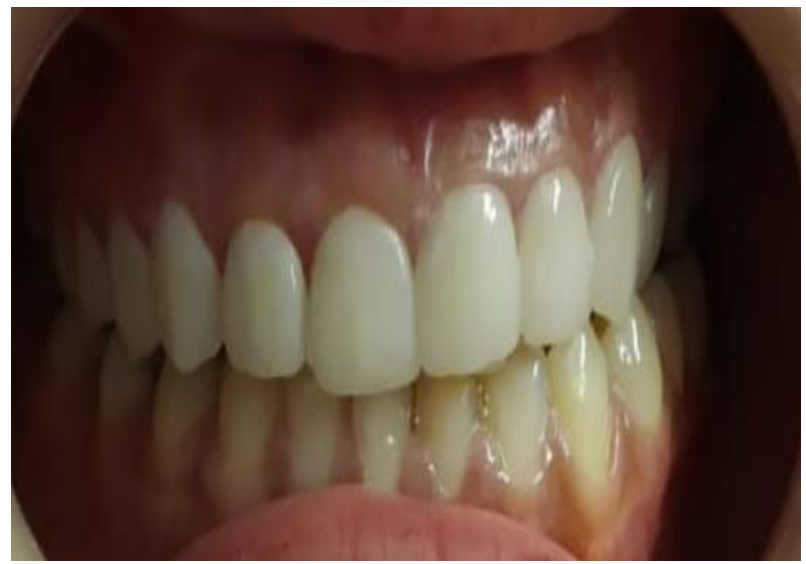

Fig. 4: Postoperative view

\section{Discussion}

The presence of compromised esthetics and spacing in the anterior tooth affect the confidence of young patients and limited their smile profile. For the treatment of spacing prosthetic and conservative approaches are generally accepted because of their minimally invasive procedures. ${ }^{3}$ The techniques and materials used to fabricate porcelain laminate veneers offer satisfactory and lasting results. Porcelain laminate behaves similarly to natural teeth because of a similar modulus of elasticity to enamel.

Patient selection and acceptance is integral part for the success of porcelain laminate veneers. In the present case because of young age a conservative method of treatment i.e, Porcelain laminate veneers wasstipulated. ${ }^{7}$ Presence of normal overjet and overbite with favorable smile line and absence of parafunction and presence of sufficient enamel made porcelain laminate veneers most preferable treatment option. The advantages of these restorations are they are naturally appealing, easy to maintain, solve the cosmetic dental issues without the use of complicated invasive procedures and are biologically acceptable to the body. ${ }^{4}$

Uses of porcelain veneers include diastema closure, fractured teeth, stained and unesthetic appearance of teeth. Incisal overlap preparation provides resistance form to the preparation and also increases the surface area. ${ }^{6}$ Therefore this preparation was chosen for this case. Etching of the veneer with hydrofluoric acid was done to increase the surface area for bonding. The depth of preparation was more than $0.8-0.9 \mathrm{~mm}$ in the middle third of the tooth therefore dual cure resin was used for cementation. 
The present case report fulfill the patients desire of space closure in anterior teeth because the patient felt shy while speaking or smiling due to compromised esthetics. The procedure was minimally invasive with veneers thickness of about 0.3 to $0.5 \mathrm{~mm}$. The patient regains the confidence which was lost due to discolorations occurred by composite veneering.

\section{Conclusion}

Aesthetic restoration on the anterior teeth of the young lady was done successfully with the help of laminate veneers fabricated from ceramic reinforced lithium disilicate. The patient was advised to maintain oral hygiene and was put on periodic recall. The laminates and veneers with proper care and knowledge provide the best esthetic and function and may prove to be a permanent solution for defective esthetics.

\section{Source of funding}

None.

\section{Conflict of interest}

None.

\section{References}

1. Kurien B, Vasunni G K, Kuruniyan M S, Correya B A. Enhancing smile with laminate veneers - A Case Report, IOSR. J Dent Med Sci 2015;14(5):49-53.

2. Soni R, Vivek R. Esthetic rehabilitation by porcelain laminates- a case report. Int J Appl Dent Sci 2015;1(4):98-100.
3. Tabassum R. Porcelain Laminate Veneers- An Esthetic Bond: A Case Report. J Cont Med A Dent 2014;2(2):60-3.

4. Alkan E, Bagis B. Midline Diastema Closure with partial laminate veneers: A Case Report, BJDM 2016;20:59-62.

5. Baranwal A K. Porcelain laminate veneer technique for esthetic correction of midline diastema. 2015;2(24):1460-3.

6. Rathod A, Bhatia P, Vijayaraghavan N V, Prakash D. Esthetic rehabilitation of patient using porcelain veneers: A series of 3 case reports. IJIDS 2007;2(1):32-40.

7. Sachdeva H, Khare S, Shankar R, Kumar N, Sthapak A. Porcelain Laminates Veneers: Case Report. J Res Adv Dent 2015;4:2:16-20.

8. Reshad M, Cascione D, Magne P. Diagnostic mock-ups as an objective tool for predictable outcomes with porcelain laminate veneers in esthetically demanding patients: a clinical report. $J$ Prosthet Dent 2008;99:333-9.

9. Friedman MJ. A 15-year review of porcelain veneer failure - A clinician's observation. Compend Contin Educ Dent 1998;19(6):625.

10. Faunce FR, Myers DR. Laminate veneer restoration of permanent incisors. J Am Dent Assoc 1976; 93:790-2.

11. Walls Awg, Steele JG, Wassell RW. Crowns and Extra Coronal Restorations: Porcelain Laminate Veneers. BDJ 2002;193(2):73-82.

How to cite this article: Mushtaq $\mathrm{H}$, Kumar $\mathrm{N}$. Enchanting smile with laminate veneers (Contact lens of teeth) - A case report. Int J Oral Health Dent 2019;5(3):151-3. 